Article

\title{
Surface Evolution of Nano-Textured 4H-SiC Homoepitaxial Layers after High Temperature Treatments: Morphology Characterization and Graphene Growth
}

\author{
Xingfang Liu ${ }^{1, *}$, Yu Chen ${ }^{2, *}$, Changzheng Sun ${ }^{3}$, Min Guan ${ }^{1}$, Yang Zhang ${ }^{1}$, Feng Zhang ${ }^{1}$, \\ Guosheng Sun ${ }^{1}$ and Yiping Zeng ${ }^{1,2}$
}

1 Key Laboratory of Semiconductor Materials Science, Institute of Semiconductors, Chinese Academy of Sciences, P.O. Box 912, Beijing 100083, China; E-Mails: guanmin@semi.ac.cn (M.G.); zhang_yang@semi.ac.cn (Y.Z.); fzhang@semi.ac.cn (F.Z.); gshsun@semi.ac.cn (G.S.); ypzeng@semi.ac.cn (Y.Z.)

2 Semiconductor Lighting Technology Research and Development Center, Institute of Semiconductors, Chinese Academy of Sciences, P.O. Box 912, Beijing 100083, China

3 Tsinghua National Laboratory for Information Science and Technology, Tsinghua University, Beijing 100084, China; E-Mail: czsun@tsinghua.edu.cn

* Authors to whom correspondence should be addressed; E-Mails: liuxf@mail.semi.ac.cn (X.L.); chenyu@semi.ac.cn (Y.C.); Tel.: +86-10-8230-4101 (X.L.), +86-10-8230-4783 (Y.C.); Fax: +86-10-8230-4232 (X.L. \& Y.C.).

Academic Editor: Thomas Nann

Received: 5 August 2015 / Accepted: 11 September 2015 / Published: 18 September 2015

\begin{abstract}
Nano-textured 4H-SiC homoepitaxial layers (NSiCLs) were grown on $4 \mathrm{H}-\mathrm{SiC}(0001)$ substrates using a low pressure chemical vapor deposition technique (LPCVD), and subsequently were subjected to high temperature treatments (HTTs) for investigation of their surface morphology evolution and graphene growth. It was found that continuously distributed nano-scale patterns formed on NSiCLs which were about submicrons in-plane and about 100 nanometers out-of-plane in size. After HTTs under vacuum, pattern sizes reduced, and the sizes of the remains were inversely proportional to the treatment time. Referring to Raman spectra, the establishment of multi-layer graphene (MLG) on NSiCL surfaces was observed. MLG with $s p^{2}$ disorders was obtained from NSiCLs after a high temperature treatment under vacuum at $1700 \mathrm{~K}$ for two hours, while MLG without $s p^{2}$ disorders was obtained under Ar atmosphere at $1900 \mathrm{~K}$.
\end{abstract}


Keywords: nano-textured; 4H-SiC; morphology; graphene; evolution

\section{Introduction}

Silicon carbide ( $\mathrm{SiC}$ )-derived carbon, especially graphene, which can be grown on $\mathrm{SiC}$ by thermal decomposition at high temperature under vacuum [1], has attracted intense interest in recent years. Graphene is a kind of planar honeycomb structure, formed by carbon atoms by means of $s p^{2}$ hybrid chemical bonds, whose unique electronic properties have been predicted in theory for decades [2]. Since graphene has been prepared experimentally [3-9], many of its properties have been verified [10-13].

Graphene preparation via high temperature treatments (HTTs) possesses at least two merits. First, thermal decomposition is a relatively simple process, since it can be performed at a wide temperature range, e.g., 1400 2000 K under high or medium vacuum, even at Ar ambient conditions [7]. Second, after graphene growth, the $\mathrm{SiC}$ host can be used as the substrate for graphene device fabrication, thus avoiding film assembly and/or transfer as necessitated by graphene production methods without substrate, via solution [14] or mechanical exfoliation [3,15]. In early stages, semi-insulating hexagonal $\mathrm{SiC}$ substrates without miss-cut surfaces, i.e., on-axis surfaces, were chosen for graphene growth; this choice was determined by the fact that commercially available, semi-insulating substrates may thus be used for electronic isolation, while continuous graphene film growth is anticipated with on-axis substrates but not with miss-cut ones, since there are steps on the surfaces of the miss-cut substrates [16] which perhaps make graphene film discontinuous. In order to explore new perspectives for graphene sciences, n-type, hexagonal $\mathrm{SiC}$ films with miss-cut surfaces [17,18] and cubic SiC films, grown heteroepitaxially on silicon substrates $[19,20]$, were also investigated.

In addition to growth on flat, rigid substrates to facilitate the planar device fabrication process, methods have been found to combine graphene with nano-materials, forming nanostructures which are expected for use in potential applications in nanoelectronics and optoelectronics [21]. Although much research on graphene growth has been performed on flat $\mathrm{SiC}$ surfaces [22,23], structured $\mathrm{SiC}$ surfaces [24], and discrete SiC particles [25], there is a dearth of research attending to surface evolution of nano-textured patterns on rigid $\mathrm{SiC}$ after high temperature treatments. For the first time, we reveal surface evolution and graphene growth aspects of nano-textured homoepitaxial $4 \mathrm{H}-\mathrm{SiC}$ layers (NSiCLs) after high temperature treatments. These results will be useful for future nano, SiC-derived graphene growth.

\section{Results and Discussion}

Due to high density nano-terraces on the epitaxial surfaces, which guide epitaxial growth in the so-called "step-control growth mechanism", 4H-SiC homoepitaxial layers with smooth and uniform morphologies are usually grown on $4 \mathrm{H}(0001)$ substrates by low pressure chemical vapor deposition (LPCVD) [26,27]. According to the said mechanism, homoepitaxial growth occurs in two-dimensional (2D) mode, while the homoepitaxial layers grow on the substrate layer by layer, often yielding a moderate growth rate of $1-2 \mu \mathrm{m} / \mathrm{h}$. However, when precursor flow increases, the growth rate rises to $40-120 \mu \mathrm{m} / \mathrm{h}$ for fast growth, disrupting the step-control growth mechanism, and often yielding epitaxial 
layers of rough morphology with microstructures [28]. At fast growth mode, there are plenty of SiC species simultaneously reaching their growth sites, usually the kinks in the terraces, which leads to disordered terrace growth. When terraces are discrete and tilted with each other at the start of epitaxial growth, nano-textures form whose feature sizes increase with the increased thickness of the epitaxial layer. Figure 1a shows the atomic force microscopy (AFM) morphology of a nano-textured, $4 \mathrm{H}-\mathrm{SiC}$ homepitaxial layer. Although the surface of the epitaxial layer appears mirror-like under Nomarski optical microscopy, it is obvious to see that there are nano-features on the surface. AFM morphologies of NSiCLs differ from those of homoepitaxial layers grown by conventional configuration with a moderate growth rate; the latter often show no features, or show wide terraces which are almost parallel with each other [29]. The AFM morphologies of NSiCLs also differ from those of thick homoepitaxial layers grown in fast mode; the surfaces of thick layers are often rough, with terraces bunched and disordered [28].
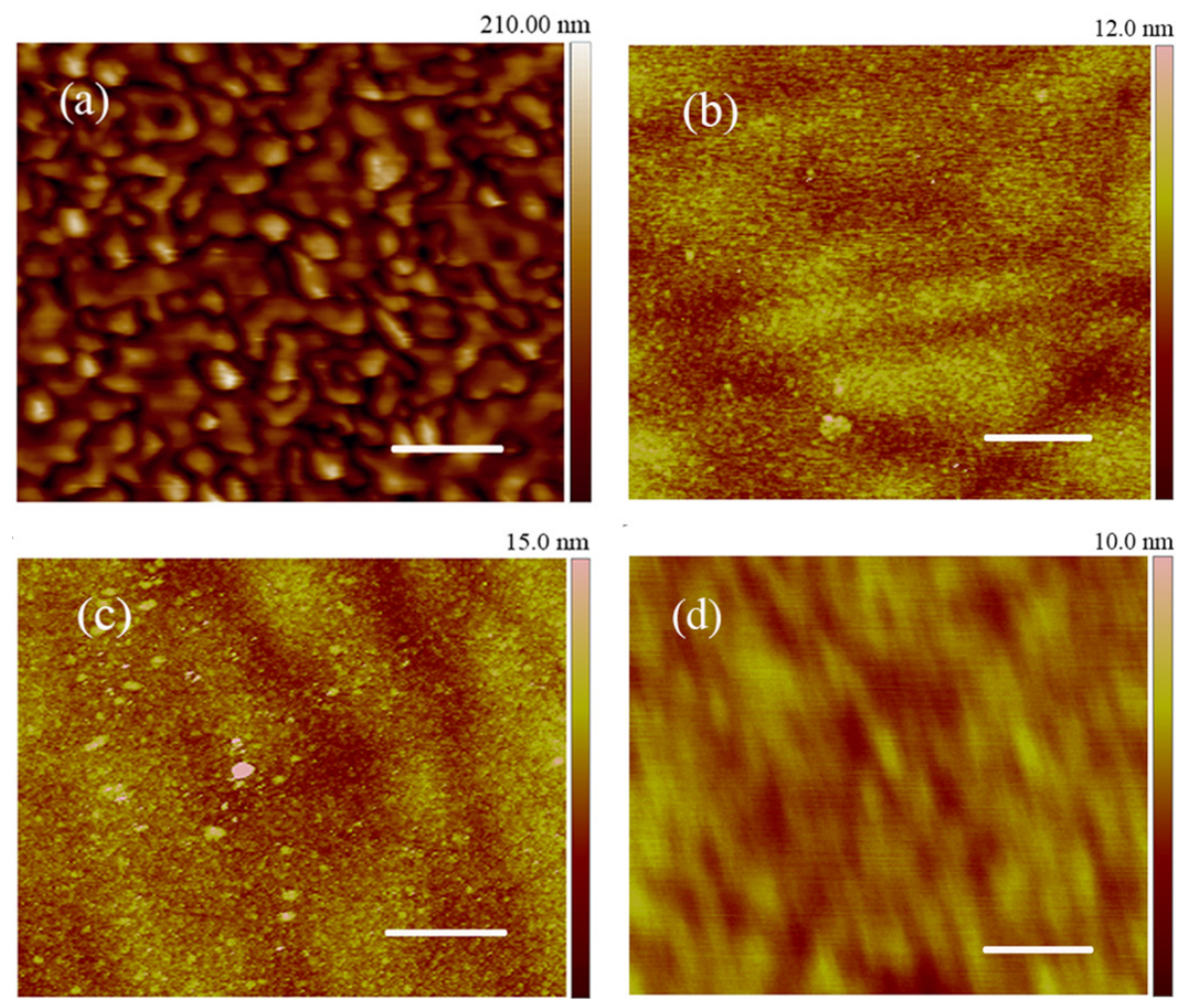

Figure 1. AFM morphologies of (a) NsiCL (Nano-textured 4H-SiC homoepitaxial layers), graphene (b) G-1h-1700K, (c) G-2h-1700K and (d) G-1h-1900K. Scale bars: $2 \mu \mathrm{m}$.

Nano-textures evolve after high temperature treatments. Figure 1b,c displays AFM morphologies of samples G-1h-1700K and G-2h-1700K, treated at $1700 \mathrm{~K}$ under medium vacuum of $10^{-3} \mathrm{~Pa}$ for one hour and two hours, respectively (please find sample specifics in the experimental section). Nano-features still appear on the surfaces of the mentioned samples; however, sizes of the features are miniscule compared to those of NSiCLs. Although binary compound $\mathrm{SiC}$ is chemically inert and it is difficult to break the $\mathrm{Si}-\mathrm{C}$ bonds, when subjected to high temperature treatment, silicon and carbon bonds break from surface level to a few monolayers beneath, and silicon atoms detach from the SiC surface while the remaining carbon atoms rebond with each other and rearrange on the surface. Nano-texture surfaces will be more active for decomposition than flat surfaces due to their larger surface area. They are also especially 
reactive on the edges since there are more unsaturated bonds and fewer bonds that have to break to detach an atom from an edge. At high temperature, those $\mathrm{Si}-\mathrm{C}$ bonds at lower-energy barriers will break more easily, while unbound $\mathrm{SiC}$ molecules or clusters will detach from surfaces until all lower-energy barrier bonds are broken. In the end, nano-texture surfaces will lose many SiC fractures, becoming smoother with smaller features, as shown in Figure 1b-d. Figure 1d depicts the AFM morphology of sample G-1h-1900K, treated at $1900 \mathrm{~K}$ under Ar atmosphere, whose morphology is stripe-like, different from those of G-1h-1700K and G-2h-1700K, which are grainy and similar to NSiCLs.

Feature sizes of those samples and their evolution are illustrated in Figure 2. NSiCLs develop at about micron scale in-plane and at nano scale out-of-plane, all of which can be roughly divided into 12 categories, labeled $1-12$ in sizes ranging from 10 to 290 (the unit is $10^{-3}$ square microns, as it is below), as shown in Figure 2a. The most miniscule category (labeled 1) comprises the largest sample population at about $40 \%$. Categories $2-7$, with sizes $30 \sim 160$, are evenly distributed, containing a collective $50 \%$ of total samples, while the remaining $10 \%$ are evenly distributed among the remaining categories. To describe this distribution, the feature sizes of NSiCL can be fitted to a three-stage, exponential decay curve, $f(x)$, expressed as:

$$
f(x)=a_{0}+\sum_{i=1}^{3} a_{i} e^{-\left(x-x_{0}\right) / t_{i}}
$$

where $a_{0}, a_{i}, t_{i}$, and $x_{0}$ are fitting constants, and the variable parameter $x$ is the in-plane lateral position.

Apart from feature sizes, the morphologies of graphene G-1h-1700K and G-2h-1700K are similar to each other and to that of NSiCL (Figure 1). We chose graphene G-2h-1700K (the corresponding AFM image is Figure 1c) as the sample for feature size evaluation. The feature sizes of graphene occur reduced by nearly two orders of magnitude (Figure $2 b$ ) when compared to their corresponding raw NSiCLs (Figure 2a), while the appropriate number of categories is also reduced to eight. A three-stage, exponential decay curve similar to the formula in Equation (1) can be used to fit the population data. The first stage (labeled 1 ) is the category with plane feature sizes of about $0.2-0.3$, which correspond to more than $70 \%$ of samples, while the second stage comprises only one category (labeled 2) holding about $10 \%$ of samples. The final stage, containing categories labeled $3-8$, is also evenly-distributed, and possesses less than $20 \%$ of samples.

To further investigate the in-plane and out-of-plane feature sizes of NSiCL and graphene, we performed line-sectional analyses on AFM images. Using a horizontal line across the middle of the images, sectional data were extracted from AFM images in Figure 1, where the undulating topographies of NSiCL and graphene with position changes are clearly shown in Figure 2c-f. The in-plane feature sizes are consistent with results from the statistical distribution analyses (Figure 2a,b), while the out-of-plane feature sizes of NSiCL and graphene are significantly different. The former are in a range of $20 \mathrm{~nm}-140$ $\mathrm{nm}$, while the latter only several nanometers. The amplitude of the out-of-plane graphene features is so small that they mostly form a straight line on the sectional analysis graph when compared to that of the NSiCL (Figure 2c). The feature size profiles of graphene G-1h-1700K and G-2h-1700K are almost the same, except that the amplitude of the out-of-plane feature sizes of G-1h-1700K (Figure 2d) is slightly smaller than those of G-2h-1700K (Figure 2e). Compared to the rough profile of G-1h-1700K (or G-2h-1700K), which is composed of many zig-zag curves forming an undulating outline, the profile of G-1h-1900K is smoother, with each outline composed of none to a few zig-zag curves (Figure $2 \mathrm{f}$ ). 

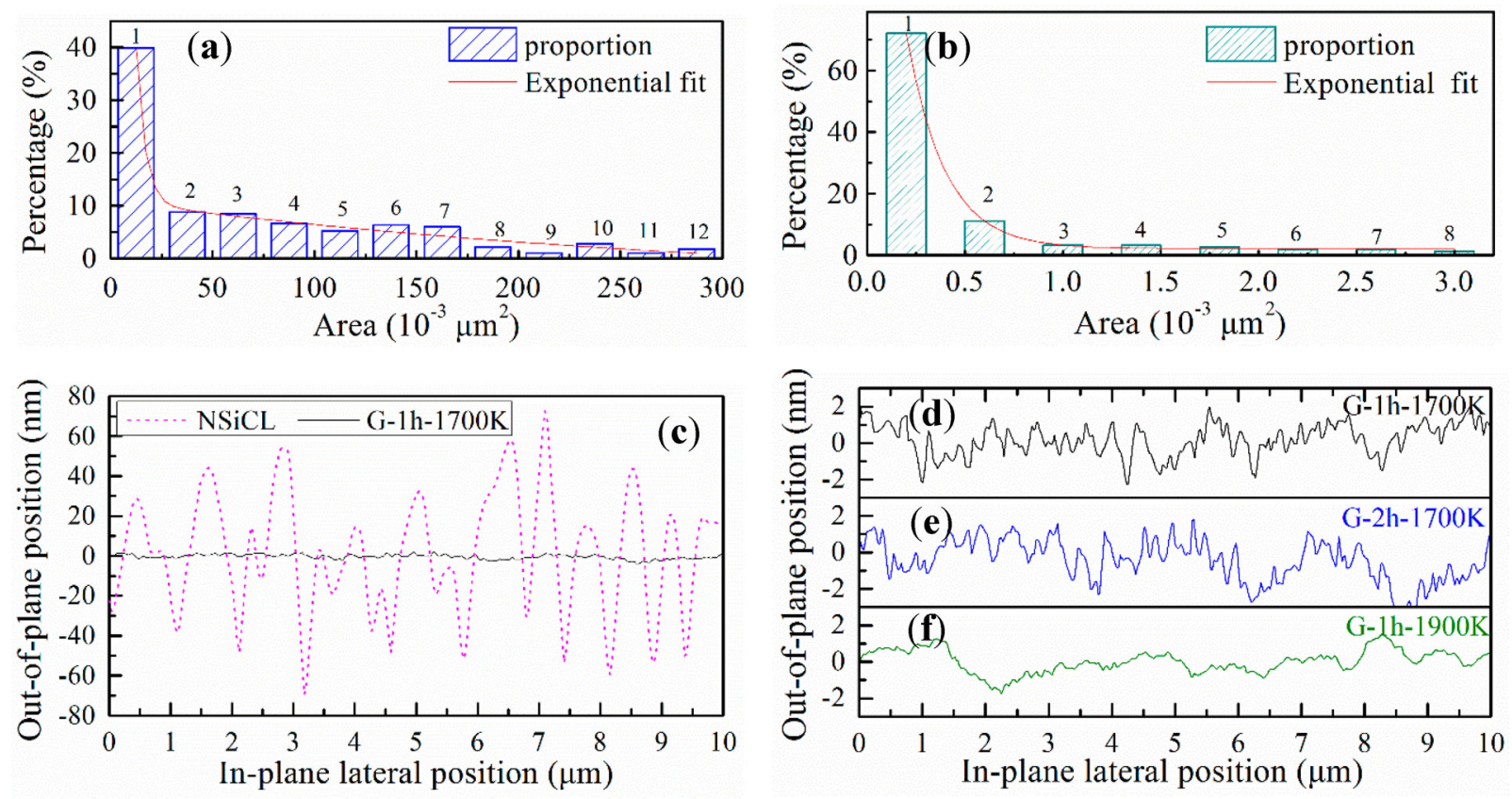

Figure 2. Statistical histograms of (a) NSiCL and (b) graphene G-2h-1700K, and line-sectional analyses of (c) NSiCL and graphene G-1h-1700K, (d) G-1h-1700K, (e) G-2h-1700K and (f) G-1h-1900K.

We used Raman spectra to judge whether NSiCL is a $4 \mathrm{H}$ polytype, as well as find layer and defect information of the obtained graphene. Figure 3 shows respective Raman spectra of as-grown NSiCL and NSiCL subjected to high temperature treatment, respectively, and the derived graphene (sample G-2h-1700K). Judging from all the Raman spectra, the as-grown NSiCLs are the 4H polytype, as indicated by Raman peaks of the transverse optic (TO) mode and longitudinal optic (LO) mode, wholly belonging to the $4 \mathrm{H}$ polytype [30], while no other peak appears, such as the $3 \mathrm{C}$ polytype. After high temperature treatment, the Raman spectrum of NSiCL has three additional peaks in the range of $1200 \mathrm{~cm}^{-1}-2800 \mathrm{~cm}^{-1}$. Usually named D mode, G mode, and 2D mode, respectively, peaks located at $1350 \mathrm{~cm}^{-1}, 1580 \mathrm{~cm}^{-1}$, and $2700 \mathrm{~cm}^{-1}$ relate to graphene signals [31], which indicate that graphene has been obtained from NSiCL after high temperature treatment. To clearly illustrate the Raman spectrum of graphene, the SiC Raman signals are often subtracted [32].

Figure 4 shows D modes, G modes, and 2D modes of Raman spectra of graphene G-1h-1700K, G-2h-1700K, and G-1h-1900K, respectively, where NSiCL Raman signals were subtracted before graphene Raman spectra composition [32]. All named Raman peaks of G-1h-1700K appear but are barely distinguishable; we are therefore not sure if, or to what extent, graphene was grown on NSiCL in this case. It is more practical to speculate that graphene growth initiates at this growth condition (Figure 5a), and that graphene will grow on NSiCL with increased growth time. In the case of G-2h-1700K, we increased only growth time, while keeping other growth conditions the same as those of G-1h-1700K, and graphene was indeed grown on NSiCL (Figure 5b).

The 2D mode is the fingerprint signal by which graphene can be identified [31]. The frequency intensity ratio of the $2 \mathrm{D}$-to-G peak (denoted as $I_{2 \mathrm{D}} / I_{\mathrm{G}}$ ) is used to identify the number of graphene layers [33]. Raman spectra with $I_{2 \mathrm{D}} / I_{\mathrm{G}} \sim 2$ indicate monolayer graphene, while those with $1<I_{2 \mathrm{D}} / I_{\mathrm{G}}<2$ 
indicate bilayer graphene [6]. The values of $I_{2 \mathrm{D}} / I_{\mathrm{G}}$ for our samples are all less than 1 , indicating that the obtained graphene are multi-layer graphene (MLG) (Figure 5c).

The full width at half maximum (FWHM) of the 2D peak is another indicator of graphene quality [34]. G-2h-1700K shows a symmetrical 2D peak, which appears at $2702 \mathrm{~cm}^{-1}$, plus the 2D peak can be fitted by a one-peak Lorentz fitting curve (Figure 4c). For a single layer of graphene, the cut-off FWHM of the 2D peak is $30 \mathrm{~cm}^{-1}$ [35]. The 2D FWHM increases with an increased number from mono-layer to multi-layer. For bilayer graphene, the 2D FWHM is found to be $43-53 \mathrm{~cm}^{-1}$. For trilayer graphene, the 2D FWHM is found to be $56-63 \mathrm{~cm}^{-1}$ [36]. The 2D FWHM of G-2h-1700K is $61 \mathrm{~cm}^{-1}$, obtained from the Lorentz fitting curve. Judging from the above analyses, G-2h-1700K is trilayer graphene. However, the Raman peaks of G-1h-1700K and G-1h-1900K are not readily suitable for Lorentz fitting. G-1h-1700K is an instance of initial graphene growth; the Raman peaks of it are barely distinguishable, so the fitted values are not reliable. G-1h-1900K displays a more complex peak than G-2h-1700K, and the $2 \mathrm{D}$ peak of G-1h-1900K is asymmetrical with an attendant peak located at $2784 \mathrm{~cm}^{-1}$. We speculate the possible origin of this shape is correlated with the topography of the substrate surface. For example, the Raman spectrum is collected over several nano-textured regions of the graphene surface, subjected to different amounts and types of strain, as those of Robinson et al. were reported [37]. The D peak is associated with $s p^{2}$ defects or local disorders [38]. Graphene G-2h-1700K is therefore more defective than graphene G-1h-1900K in that no D peak appears in G-1h-1900K Raman spectrum.

Figure 5 is a schematic representation of the surface evolution and graphene growth on a grain of the nano-textured $\mathrm{SiC}$ film during high temperature annealing. Here, the routes of (a), (b) and (c) in Figure 5, which stands for instances of initial growth (G-1h-1700K), disordered graphene growth (G-2h-1700K), and multi-layer graphene growth (G-1h-1900K), respectively, according to various treatment techniques. This schematic includes interpretations for each of our samples.

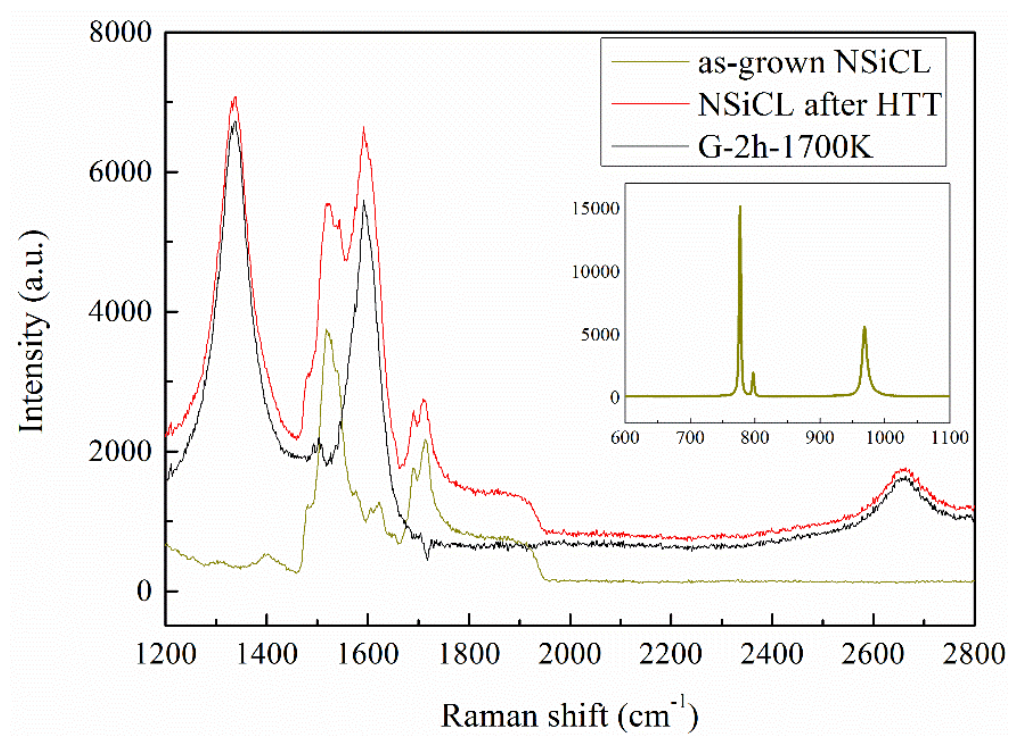

Figure 3. Raman spectra of the as-grown NSiCL and the NSiCL after a high temperature treatment (HTT), respectively, and the derived graphene of sample G-2h-1700K. Inset: Raman peaks of TO mode and LO mode of the as-grown NSiCL. 


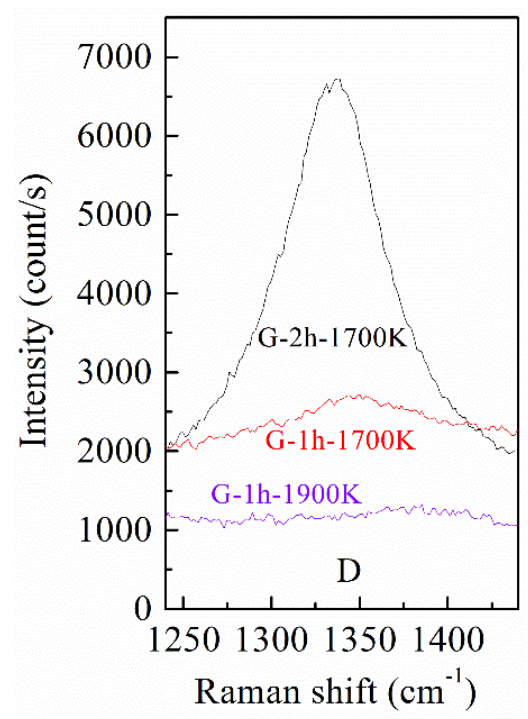

(a)

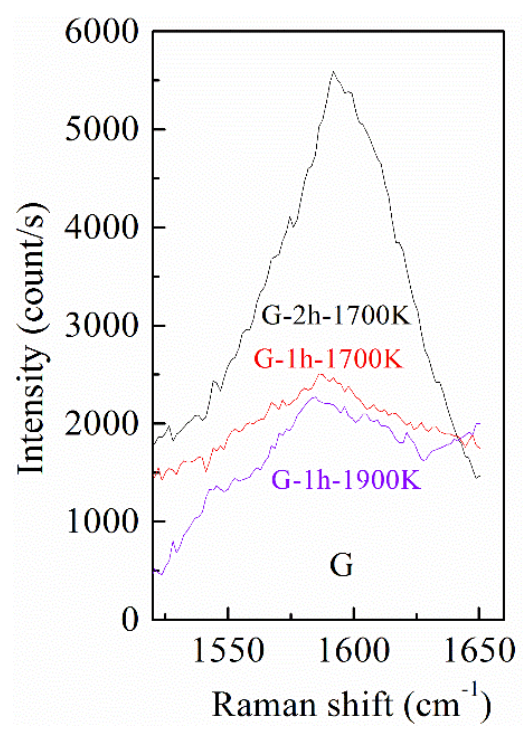

(b)

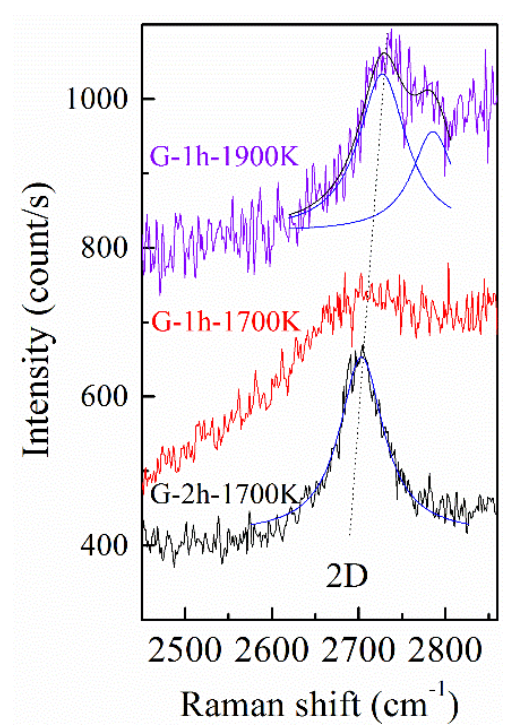

(c)

Figure 4. Raman spectra of graphene G-1h-1700K, G-2h-1700K, and G-1h-1900K: (a) D peaks, (b) G peaks and (c) 2D peaks.

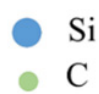

C

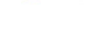

(a)

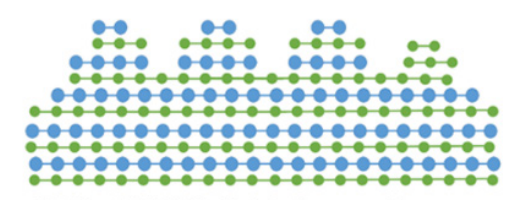

G-1h-1700K: initial growth

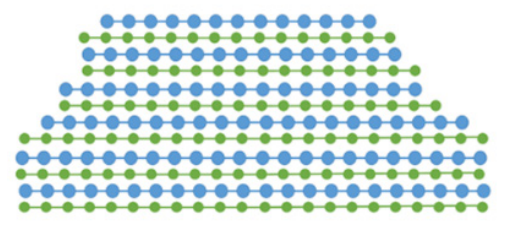

NSiCL (one grain) (b)

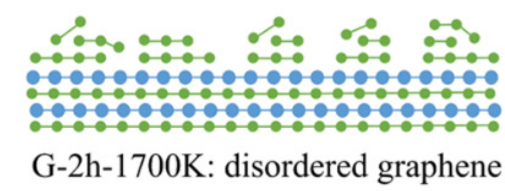

(c)

Figure 5. Schematic illustration for surface evolution of NSiCL (one grain extracted from the nano-textured $\mathrm{SiC}$ layer as an example) and corresponding graphene growth. After subjection to high temperature treatments (HTTs), nano-patterns on the SiC layer were reduced. Here, (a) G-1h-1700K; (b) G-2h-1700K; and (c) G-1h-1900K are, respectively, instances of initial growth, disordered graphene growth, and multi-layer graphene growth according to various treatment techniques.

When graphene is grown on the $\mathrm{Si}$-face of $\mathrm{SiC}(0001)$, it often forms an interfacial buffer layer of carbon between the Si face and graphene [39]. This is believed to be at the origin of the Bernal stacking in multi-layer graphene on the Si face. Such a buffer layer is not present for other SiC orientations, i.e., the C-face [40] and the non-polar faces [41]. Furthermore, it has been recently shown that the buffer 
layer is locally delaminated from oblique facets $(11 \overline{2} \mathrm{n})$ of off-angle Si-face SiC wafers [42]. In this study, graphene has been grown on the Si-face of $\mathrm{SiC}(0001)$, hence it would form an interfacial buffer layer; however, the SiC surface is nano-textured, with oblique facets $(11 \overline{2} \mathrm{n})$, and, therefore, the buffer layer is locally delaminated [42]. This result is different than in previous studies $[4,7,43]$, in which flat $4 \mathrm{H}-\mathrm{SiC}$ substrates were used.

\section{Experimental Section}

Nano-textured 4H-SiC homoepitaxial layers (NSiCLs) with nominal thickness and doping concentration of $620 \mathrm{~nm}$ and $5.8 \times 10^{15} \mathrm{~cm}^{-3}$, respectively, were grown on commercially available, on-axis $4 \mathrm{H}-\mathrm{SiC}(0001)$ substrates, using low pressure chemical vapor deposition. In order to obtain surfaces with nano-textured morphology, an unconventional growth technique was adopted. Before epitaxial growth, $4 \mathrm{H}-\mathrm{SiC}(0001)$ substrates were etched under $\mathrm{H}_{2}$ atmosphere at $1600 \mathrm{~K}$ for one hour to prepare clean and smooth surfaces. After etching, the temperature was raised to $1850 \mathrm{~K}$ rapidly, while $\mathrm{SiHCl}_{3}$ and $\mathrm{C}_{2} \mathrm{H}_{4}$ as precursors, diluted in highly pure $\mathrm{H}_{2}(99.999 \%)$, were fed into the chemical vapor deposition (CVD) chamber for epitaxial growth. Precursors were turned off after a $30 \mathrm{~s}$ growth period, and the $4 \mathrm{H}-\mathrm{SiC}$ samples cooled down to room temperature naturally under $\mathrm{H}_{2}$ atmosphere. Pressures were kept at $10^{4} \mathrm{~Pa}$ during the whole process.

NSiCLs were subjected to high temperature treatments in an induction furnace. Samples were treated at $1700 \mathrm{~K}$ under medium vacuum of $10^{-3} \mathrm{~Pa}$ for one and two hours, respectively, while some samples were treated at $1900 \mathrm{~K}$ under Ar atmosphere of $1.013 \times 10^{5} \mathrm{~Pa}(1 \mathrm{~atm})$ for one hour. The corresponding samples are denoted as G-1h-1700K, G-2h-1700K, and G-1h-1900K, respectively.

Morphologies of both NSiCL and graphene were characterized by atomic force microscopy (AFM, Veeco Instruments, NewYork, NY, USA), while morphology features were characterized by statistical histogram and sectional analyses. Raman scattering spectra (Horiba HR800, Horiba Jobin Yvon, Paris, France) were used to evaluate graphene quality, and were performed at room temperature using an He-Ne laser operating at the $632.8 \mathrm{~nm}$ line mode with a confocal microscope at about $1 \mu \mathrm{m}$ spatial resolution.

\section{Conclusions}

In summary, nano-textured $4 \mathrm{H}-\mathrm{SiC}$ homoepitaxial layers (NSiCLs) were grown on $4 \mathrm{H}-\mathrm{SiC}(0001)$ by an unconventional epitaxial method using low pressure chemical vapor deposition, producing as-grown NSiCLs with feature sizes of about 0.01-0.29 square microns. After subjection to different high temperature treatments, feature sizes of the NSiCLs were reduced, while remaining features were at least two orders of magnitude smaller than on as-grown NSiCLs. Graphene was grown on these $4 \mathrm{H}-\mathrm{SiC}$ homoepitaxial layers. The initial growth of graphene took place after a high temperature treatment at $1700 \mathrm{~K}$ under medium vacuum of $10^{-3} \mathrm{~Pa}$ for one hour, while multi-layer graphene (MLG) grew in two hours. MLG without $s p^{2}$ disorders was also grown under argon once NSiCL was treated at the higher temperature of $1900 \mathrm{~K}$. This investigation produces an avenue of refined technique for graphene production, expanding the pathway for future nano, SiC-derived graphene growth. 


\section{Acknowledgments}

This work was supported by the National Natural Science Foundation of China (grant No. 61274007, 61274008, 61274049, 61376071, 61474113 and 61475151), the Beijing Natural Science Foundation (grant No. 4132070, 4132074 and 4132076) and the Tsinghua National Laboratory for Information Science and Technology (NLIST) Cross-discipline Foundation (grant No. 42003175 and 420003179).

\section{Author Contributions}

The study was designed by Xingfang Liu and Yu Chen. The experiments were performed by Xingfang Liu and Yang Zhang. The data analysis was performed by Xingfang Liu, Yu Chen, Changzheng Sun, Min Guan, Feng Zhang, Guosheng Sun, and Yiping Zeng. The manuscript was verified by all authors.

\section{Conflicts of Interest}

The authors declare no conflict of interest.

\section{References}

1. Berger, C.; Song, Z.M.; Li, T.B.; Li, X.B.; Ogbazghi, A.Y.; Feng, R.; Dai, Z.T.; Marchenkov, A.N.; Conrad, E.H.; First, P.N.; et al. Ultrathin epitaxial graphite: 2D electron gas properties and a route toward graphene-based nanoelectronics. J. Phys. Chem. B 2004, 108, 19912-19916.

2. Gonzalez, J.; Guinea, F.; Vozmediano, M.A.H. Electron-electron interactions in graphene sheets. Phys. Rev. B 2001, 6313, 134421.

3. Novoselov, K.S.; Geim, A.K.; Morozov, S.V.; Jiang, D.; Zhang, Y.; Dubonos, S.V.; Grigorieva, I.V.; Firsov, A.A. Electric field effect in atomically thin carbon films. Science 2004, 306, 666-669.

4. Berger, C.; Song, Z.; Li, X.; Wu, X.; Brown, N.; Naud, C.; Mayou, D.; Li, T.; Hass, J.; Marchenkov, A.N.; et al. Electronic confinement and coherence in patterned epitaxial graphene. Science 2006, 312, 1191-1196.

5. Kim, K.S.; Zhao, Y.; Jang, H.; Lee, S.Y.; Kim, J.M.; Kim, K.S.; Ahn, J.-H.; Kim, P.; Choi, J.-Y.; Hong, B.H. Large-scale pattern growth of graphene films for stretchable transparent electrodes. Nature 2009, 457, 706-710.

6. Li, X.; Cai, W.; An, J.; Kim, S.; Nah, J.; Yang, D.; Piner, R.; Velamakanni, A.; Jung, I.; Tutuc, E.; et al. Large-Area Synthesis of High-Quality and Uniform Graphene Films on Copper Foils. Science 2009, 324, 1312-1314.

7. Emtsev, K.V.; Bostwick, A.; Horn, K.; Jobst, J.; Kellogg, G.L.; Ley, L.; McChesney, J.L.; Ohta, T.; Reshanov, S.A.; Rohrl, J.; et al. Towards wafer-size graphene layers by atmospheric pressure graphitization of silicon carbide. Nat. Mater. 2009, 8, 203-207.

8. Cai, T.; Jia, Z.; Yan, B.; Yu, D.; Wu, X. Hydrogen assisted growth of high quality epitaxial graphene on the C-face of 4H-SiC. Appl. Phys. Lett. 2015, 106, 013106.

9. Adan-Mas, A.; Wei, D. Photoelectrochemical Properties of Graphene and Its Derivatives. Nanomaterials 2013, 3, 325-356. 
10. Tedesco, J.L.; VanMil, B.L.; Myers-Ward, R.L.; McCrate, J.M.; Kitt, S.A.; Campbell, P.M.; Jernigan, G.G.; Culbertson, J.C.; Eddy, C.R.; Gaskill, D.K. Hall effect mobility of epitaxial graphene grown on silicon carbide. Appl. Phys. Lett. 2009, 95, 122102.

11. Cummings, A.W.; Duong, D.L.; Nguyen, V.L.; Dinh Van, T.; Kotakoski, J.; Barrios, V.J.E.; Lee, Y.H.; Roche, S. Charge Transport in Polycrystalline Graphene: Challenges and Opportunities. Adv. Mater. 2014, 26, 5079-5094.

12. Kumari, A.; Prasad, N.; Bhatnagar, P.K.; Mathur, P.C.; Yadav, A.K.; Tomy, C.V.; Bhatia, C.S. Electrical transport properties of polycrystalline CVD graphene on $\mathrm{SiO}_{2} / \mathrm{Si}$ substrate. Diam. Relat. Mater. 2014, 45, 28-33.

13. Lin, Y.M.; Dimitrakopoulos, C.; Jenkins, K.A.; Farmer, D.B.; Chiu, H.Y.; Grill, A.; Avouris, P. 100-GHz Transistors from Wafer-Scale Epitaxial Graphene. Science 2010, 327, 662-662.

14. Tung, V.C.; Allen, M.J.; Yang, Y.; Kaner, R.B. High-throughput solution processing of large-scale graphene. Nat. Nanotechnol. 2009, 4, 25-29.

15. Yi, M.; Shen, Z. A review on mechanical exfoliation for the scalable production of graphene. J. Mater. Chem. A 2015, 3, 11700-11715.

16. Grodecki, K.; Bozek, R.; Strupinski, W.; Wysmolek, A.; Stepniewski, R.; Baranowski, J.M. Micro-Raman spectroscopy of graphene grown on stepped 4H-SiC (0001) surface. Appl. Phys. Lett. 2012, 100, 261604.

17. Ouerghi, A.; Silly, M.G.; Marangolo, M.; Mathieu, C.; Eddrief, M.; Picher, M.; Sirotti, F.; el Moussaoui, S.; Belkhou, R. Large-Area and High-Quality Epitaxial Graphene on Off-Axis SiC Wafers. ACS Nano 2012, 6, 6075-6082.

18. Camara, N.; Jouault, B.; Caboni, A.; Jabakhanji, B.; Desrat, W.; Pausas, E.; Consejo, C.; Mestres, N.; Godignon, P.; Camassel, J. Growth of monolayer graphene on 8 degrees off-axis 4H-SiC (000-1) substrates with application to quantum transport devices. Appl. Phys. Lett. 2010, $97,093107$.

19. Ouerghi, A.; Balan, A.; Castelli, C.; Picher, M.; Belkhou, R.; Eddrief, M.; Silly, M.G.; Marangolo, M.; Shukla, A.; Sirotti, F. Epitaxial graphene on single domain 3C-SiC(100) thin films grown on off-axis Si(100). Appl. Phys. Lett. 2012, 101, 021603.

20. Gupta, B.; Notarianni, M.; Mishra, N.; Shafiei, M.; Iacopi, F.; Motta, N. Evolution of epitaxial graphene layers on $3 \mathrm{C} \mathrm{SiC/Si} \mathrm{(111)} \mathrm{as} \mathrm{a} \mathrm{function} \mathrm{of} \mathrm{annealing} \mathrm{temperature} \mathrm{in} \mathrm{UHV.} \mathrm{Carbon} \mathrm{2014,}$ 68, 563-572.

21. Safron, N.S.; Kim, M.; Gopalan, P.; Arnold, M.S. Barrier-Guided Growth of Micro- and Nano-Structured Graphene. Adv. Mater. 2012, 24, 1041-1045.

22. Dharmaraj, P.; Jeganathan, K.; Parthiban, S.; Kwon, J.Y.; Gautam, S.; Chae, K.H.; Asokan, K. Selective area growth of bernal bilayer epitaxial graphene on $4 \mathrm{H}-\mathrm{SiC}$ (0001) substrate by electron-beam irradiation. Appl. Phys. Lett. 2014, 105, 181601.

23. Pallecchi, E.; Lafont, F.; Cavaliere, V.; Schopfer, F.; Mailly, D.; Poirier, W.; Ouerghi, A. High Electron Mobility in Epitaxial Graphene on $4 \mathrm{H}-\mathrm{SiC}(0001)$ via post-growth annealing under hydrogen. Sci. Rep. 2014, 4, 4558.

24. Hu, Y.; Ruan, M.; Guo, Z.; Dong, R.; Palmer, J.; Hankinson, J.; Berger, C.; Heer, W.A.D. Structured Epitaxial Graphene: Growth and Properties. J. Phys. D Appl. Phys. 2012, 45, 154010. 
25. Zhu, K.; Guo, L.; Lin, J.; Hao, W.; Shang, J.; Jia, Y.; Chen, L.; Jin, S.; Wang, W.; Chen, X. Graphene covered SiC powder as advanced photocatalytic material. Appl. Phys. Lett. 2012, 100, 023113.

26. Dong, L.; Sun, G.; Yu, J.; Zheng, L.; Liu, X.; Zhang, F.; Yan, G.; Li, X.; Wang, Z. Growth of $4 \mathrm{H}-\mathrm{SiC}$ epilayers with low surface roughness and morphological defects density on 4 degrees off-axis substrates. Appl. Surf. Sci. 2013, 270, 301-306.

27. Itoh, A.; Akita, H.; Kimoto, T.; Matsunami, H. High-quality 4H-SiC homoepitaxial layers grown by step-controlled epitaxy. Appl. Phys. Lett. 1994, 65, 1400-1402.

28. Henry, A.; Hassan, J.; Bergman, J.P.; Hallin, C.; Janzen, E. Thick silicon carbide homoepitaxial layers grown by CVD techniques. Chem. Vap. Depos. 2006, 12, 475-482.

29. Liu, B.; Sun, G.-S.; Liu, X.-F.; Zhang, F.; Dong, L.; Zheng, L.; Yan, G.-G.; Liu, S.-B.; Zhao, W.-S.; Wang, L.; et al. Fast Homoepitaxial Growth of 4H-SiC Films on 4 Degrees Off-Axis Substrates in a $\mathrm{SiH} 4-\mathrm{C}_{2} \mathrm{H}_{4}-\mathrm{H}-2$ System. Chin. Phys. Lett. 2013, 30, 128101.

30. Nakashima, S.; Harima, H. Raman investigation of SiC polytypes. Phys. Status Solidi A 1997, 162, 39-64.

31. Ferrari, A.C.; Meyer, J.C.; Scardaci, V.; Casiraghi, C.; Lazzeri, M.; Mauri, F.; Piscanec, S.; Jiang, D.; Novoselov, K.S.; Roth, S.; et al. Raman spectrum of graphene and graphene layers. Phys. Rev. Lett. 2006, 97, 187401.

32. Rohrl, J.; Hundhausen, M.; Emtsev, K.V.; Seyller, T.; Graupner, R.; Ley, L. Raman spectra of epitaxial graphene on SiC(0001). Appl. Phys. Lett. 2008, 92, 201918.

33. Hao, Y.; Wang, Y.; Wang, L.; Ni, Z.; Wang, Z.; Wang, R.; Koo, C.K.; Shen, Z.; Thong, J.T.L. Probing Layer Number and Stacking Order of Few-Layer Graphene by Raman Spectroscopy. Small 2010, 6, 195-200.

34. Lee, D.S.; Riedl, C.; Krauss, B.; von Klitzing, K.; Starke, U.; Smet, J.H. Raman Spectra of Epitaxial Graphene on SiC and of Epitaxial Graphene Transferred to $\mathrm{SiO}_{2}$. Nano Lett. 2008, 8, 4320-4325.

35. Berciaud, S.; Ryu, S.; Brus, L.E.; Heinz, T.F. Probing the Intrinsic Properties of Exfoliated Graphene: Raman Spectroscopy of Free-Standing Monolayers. Nano Lett. 2009, 9, 346-352.

36. Lee, S.; Lee, K.; Zhong, Z., Wafer Scale Homogeneous Bilayer Graphene Films by Chemical Vapor Deposition. Nano Lett. 2010, 10, 4702-4707.

37. Robinson, J.A.; Puls, C.P.; Staley, N.E.; Stitt, J.P.; Fanton, M.A.; Emtsev, K.V.; Seyller, T.; Liu, Y. Raman Topography and Strain Uniformity of Large-Area Epitaxial Graphene. Nano Lett. 2009, 9, 964-968.

38. Shlimak, I.; Haran, A.; Zion, E.; Havdala, T.; Kaganovskii, Y.; Butenko, A.V.; Wolfson, L.; Richter, V.; Naveh, D.; Sharoni, A.; et al. Raman scattering and electrical resistance of highly disordered graphene. Phys. Rev. B 2015, 91, 045414.

39. Riedl, C.; Coletti, C.; Iwasaki, T.; Zakharov, A.A.; Starke, U. Quasi-Free-Standing Epitaxial Graphene on SiC Obtained by Hydrogen Intercalation. Phys. Rev. Lett. 2009, 103, 246804.

40. Nicotra, G.; Deretzis, I.; Scuderi, M.; Spinella, C.; Longo, P.; Yakimova, R.; Giannazzo, F.; la Magna, A. Interface disorder probed at the atomic scale for graphene grown on the $\mathrm{C}$ face of $\mathrm{SiC}$. Phys. Rev. B 2015, 91, 155411.

41. Ostler, M.; Deretzis, I.; Mammadov, S.; Giannazzo, F.; Nicotra, G.; Spinella, C.; Seyller, T.; la Magna, A. Direct growth of quasi-free-standing epitaxial graphene on nonpolar $\mathrm{SiC}$ surfaces. Phys. Rev. B 2013, 88, 085408 . 
42. Nicotra, G.; Ramasse, Q.M.; Deretzis, I.; la Magna, A.; Spinella, C.; Giannazzo, F. Delaminated Graphene at Silicon Carbide Facets: Atomic Scale Imaging and Spectroscopy. ACS Nano 2013, 7 , 3045-3052.

43. Cai, S.; Liu, Z.; Zhong, N.; Liu, S.; Liu, X. Effect of Growth Pressure on Epitaxial Graphene Grown on $4 \mathrm{H}-\mathrm{SiC}$ Substrates by Using Ethene Chemical Vapor Deposition. Materials 2015, 8, 5586-5596.

(C) 2015 by the authors; licensee MDPI, Basel, Switzerland. This article is an open access article distributed under the terms and conditions of the Creative Commons Attribution license (http://creativecommons.org/licenses/by/4.0/). 